\title{
LOCAL ERGODIC THEOREMS FOR NONCOMMUTING SEMIGROUPS
}

\author{
S. A. McGRATH
}

\begin{abstract}
Let $(X, \mu)$ be a $\sigma$-finite measure space and $L_{p}(\mu), 1<p<\infty$, the usual Banach spaces of complex-valued functions. For $k=1,2, \ldots, n$, let $\left\{T_{k}(t)\right.$ : $t>0\}$ be a strongly continuous semigroup of Dunford-Schwartz operators. If

$$
f \in R_{n-1}=\left\{g: \int_{|g|>t}|g / t|(\log |g / t|)^{n-1} d \mu<\infty \text { for all } t>0\right\},
$$

then

$$
\frac{1}{\alpha_{1} \alpha_{2} \cdots \alpha_{n}} \int_{0}^{\alpha_{n}} \cdots \int_{0}^{\alpha_{i}} T_{n}\left(t_{n}\right) \cdots T_{1}\left(t_{1}\right) f(x) d t_{1} \cdots d t_{n} \rightarrow f(x)
$$

$\mu$-a.e. as $\alpha_{1} \searrow 0, \ldots, \alpha_{n} \searrow 0$ independently. If $f \in L_{p}(\mu), 1<p<\infty$, then the limit exists in norm as well as pointwise.
\end{abstract}

Introduction. Let $(X, \mu)$ be a complete, $\sigma$-finite measure space and let $L_{p}(\mu)=$ $L_{p}(X, \mu), 1<p<\infty$, be the usual Banach spaces of complex-valued functions. Let $\{T(t): t>0\}$ be a strongly continuous semigroup of $L_{1}(\mu)$ contractions. This means that (i) $T(t+s)=T(t) T(s), s, t>0$; (ii) $\|T(t)\|_{1}<1, t>0$; (iii) $f \in L_{1}(\mu)$ implies $\|T(t) f-T(s) f\|_{1} \rightarrow 0$ as $s \rightarrow t$. We assume for simplicity that $T(0)=I$. A semigroup $\{T(t)\}$ of $L_{1}(\mu)$ contractions is a Dunford-Schwartz semigroup if $\|T(t)\|_{\infty}<1$ for all $t>0$. It is a submarkovian semigroup if each $T(t)$ is a positive operator, i.e. $f \in L_{1}^{+}(\mu)$ implies $T(t) f \in L_{1}^{+}(\mu)$ for all $t>0$. A positive $L_{1}(\mu)$ semigroup $\{P(t)\}$ is said to dominate $\{T(t)\}$ if $P(t)|f| \geqslant|T(t) f| \mu$-a.e. for $f \in$ $L_{1}(\mu)$ and $t>0$.

The strong continuity of $\{T(t)\}$ permits us to define, for $\alpha>0$ and $f \in L_{1}(\mu)$, the integral $\int_{0}^{\alpha} T(t) f d t$ as the $L_{1}$-limit of Riemann sums. A more precise definition of $\int_{0}^{\alpha} T(t) f d t$ is required to investigate the pointwise convergence of $(1 / \alpha) \int_{0}^{\alpha} T(t) f d t$. It is well known ([2], [8]) that given $f \in L_{1}(\mu)$ the vector $T(t) f$ has a scalar representation $T(t) f(x)$, defined on $R^{+} \times X$ and measurable with respect to the product measure on $R^{+} \times X$, such that $T(t) f(x)$ is in the equivalence class of $T(t) f$ for all $t>0$. This representation is unique modulo sets of product measure zero. The scalar function $T(t) f(x)$ is integrable with respect to the product measure on $R^{+} \times X$. Additionally, there is a $\mu$-null set $E(f)$, independent of $\alpha>0$, outside which $\int_{0}^{\alpha} T(t) f(x) d t$ exists and, as a function of $x$, is in the equivalence class of $\int_{0}^{\alpha} T(t) f d t$ for every $\alpha>0$. We define

Received by the editors April 10, 1979.

AMS (MOS) subject classifications (1970). Primary 47D05, 28A65.

Key words and phrases. Local ergodic theorem, noncommuting semigroups, Dunford-Schwartz operators.

() 1980 American Mathematical Society 0002-9939/80/0000-0262/\$02.25 


$$
A(T, \alpha) f(x)=\frac{1}{\alpha} \int_{0}^{\alpha} T(t) f(x) d t
$$

for all $\alpha>0$ and $f \in L_{1}(\mu)$.

In [3] N. Fava showed that if $f \in R_{n-1}$ and $\left\{T_{k}(t): t>0\right\}, k=1,2, \ldots, n$, are strongly continuous semigroups of positive Dunford-Schwartz operators, then

$$
\frac{1}{\alpha_{1} \alpha_{2} \cdots \alpha_{n}} \int_{0}^{\alpha_{n}} \cdots \int_{0}^{\alpha_{1}} T_{n}\left(t_{n}\right) \cdots T_{1}\left(t_{1}\right) f(x) d t_{1} \cdots d t_{n}
$$

converges $\mu$-a.e. to a finite limit as $\alpha_{1} \rightarrow \infty, \ldots, \alpha_{n} \rightarrow \infty$ independently. The class

$$
R_{n}=\left\{f: \int_{|f| t}|f / t|(\log |f / t|)^{n} d \mu<\infty \text { for all } t>0\right\}
$$

is a subspace of $L_{1}(\mu)+L_{\infty}(\mu)$ and satisfies $L_{1}(\mu)+L_{\infty}(\mu) \supset R_{0} \supset R_{1} \supset R_{2}$ $\supset \cdots$ Also, for any $1<p<\infty$ and $n>0, L_{p}(\mu) \subset R_{n}$. Finally, $R_{n}=$ $L\left(\log ^{+} L\right)^{n}$, for all $n>0$, when $\mu(X)<\infty$. These facts are established in [3].

In this note a local ergodic theorem is established: if $\left\{T_{k}(t)\right\}, k=1,2, \ldots, n$, are strongly continuous semigroups of Dunford-Schwartz operators and $f \in R_{n-1}$, then

$$
\frac{1}{\alpha_{1} \cdots \alpha_{n}} \int_{0}^{\alpha_{n}} \cdots \int_{0}^{\alpha_{1}} T_{n}\left(t_{n}\right) \cdots T_{1}\left(t_{1}\right) f(x) d t_{1} \cdots d t_{n} \rightarrow f(x) \quad \mu \text {-a.e. }
$$

as $\alpha_{1} \searrow 0, \ldots, \alpha_{n} \searrow 0$ independently. For notational convenience we denote the integral in (*) by $A\left(T_{n}, \alpha_{n}\right) \cdots A\left(T_{1}, \alpha_{1}\right) f(x)$. Local ergodic theorems for single $L_{1}$ contraction semigroups have been established in ([4], [5], [6], [8], [9]). T. Terrell [10] extended the local ergodic theorem for one-parameter submarkovian semigroups to the $n$-parameter case. He showed that if $f \in L_{1}(\mu)$ then

$$
\lim _{\alpha \supset 0}(1 / \alpha)^{n} \int_{0}^{\alpha} \cdots \int_{0}^{\alpha} T\left(t_{1}, \ldots, t_{n}\right) f(x) d t_{1} \cdots d t_{n}=f(x) \quad \mu \text {-a.e. }
$$

He pointed out that if it is assumed only that $f \in L_{1}(\mu)$ then (*) may fail (even if the semigroups commute).

Main results. If $\{T(t): t \geqslant 0\}$ is a strongly continuous $L_{1}(\mu)$ semigroup of Dunford-Schwartz operators then a scalar representation $T(t) f(x)$ exists for any $f \in L_{p}(\mu), 1 \leqslant p<\infty$ ([2, pp. 196-198]). However $R_{n}$ is not contained in the linear span of $\cup_{1<p<\infty} L_{p}(\mu)$ [3]. Before proving our ergodic theorem we must show that a scalar representation exists for functions in $\boldsymbol{R}_{\mathbf{0}}$.

1. Lemma. Let $(X, \mu)$ be a complete, $\sigma$-finite measure space and let $\{T(t)\}$ be a strongly continuous $L_{1}(\mu)$ contraction semigroup such that for all $t>0,\|T(t) f\|_{\infty}<$ $\|f\|_{\infty}, f \in L_{1}(\mu) \cap L_{\infty}(\mu)$. Then $\{T(t)\}$ may be extended to a Dunford-Schwartz semigroup and the domain of definition of the scalar representation of $\{T(t)\}$ may be extended from $L_{1}(\mu)$ to $L_{1}(\mu)+L_{\infty}(\mu)$.

Proof. Let $\{P(t): t \geqslant 0\}$ be a strongly continuous submarkovian semigroup which dominates $\{T(t): t>0\}$ ([4], [6]). If $f \in L_{1}^{+}(\mu)$ then $P: f \rightarrow e^{-t} P(t) f(x)$ defines a linear contraction mapping from $L_{1}(\mu)$ to $L_{1}\left(R^{+} \times X, d \rho\right)$, where $d \rho=$ $d t \times d \mu$. This mapping is a positive contraction since if $f>0 \mu$-a.e. then $P(t) f>0$ 
$\boldsymbol{\mu}$-a.e. for all $t>0$ implies $P f \geq 0 \rho$-a.e. by Fubini's theorem. Consequently $\boldsymbol{P}$ may be extended to the cone of positive measurable functions on $X$ [7]. We denote the extension of $\boldsymbol{P}$ by $\hat{\boldsymbol{P}}$.

Choose $f \in L_{\infty}^{+}(\mu)$ and let $f_{k}(k=1,2, \ldots)$ be a sequence of functions in $L_{1}^{+}(\mu) \cap L_{\infty}^{+}(\mu)$ with $f_{k}(x) \nearrow f(x) \mu$-a.e. as $k \rightarrow \infty$. Then $\hat{P} f(t, x)=$ $\lim _{k \rightarrow \infty} e^{-t} P(t) f_{k}(x)$, since $\hat{P}$ has the monotone continuity property. We have $|\hat{P} f(t, x)|<\infty \rho$-a.e. since $\|\hat{P} g\|_{\infty} \leqslant\|g\|_{\infty}$ for all $g \in L_{\infty}^{+}(\mu)$. Consequently the sequence $\left\{T(t) f_{k}(x)\right\}$ is Cauchy $\mu$-a.e. since

$$
\left|T(t) f_{k+j}(x)-T(t) f_{k}(x)\right| \leqslant\left|P(t) f_{k+j}(x)-P(t) f_{k}(x)\right|
$$

Set $\hat{T}(t) f(x)=\lim _{k \rightarrow \infty} T(t) f_{k}(x), f \in L_{\infty}^{+}(\mu)$. It is not difficult to show that our definition is independent, modulo $\rho$-null sets, of the particular sequence $\left\{f_{k}\right\}$ converging to $f$. Extend now $\hat{T}(t) f(x)$ to $L_{\infty}(\mu)$ by linearity. If we set $T(t) f=$ $\hat{T}(t) f(\cdot), f \in L_{\infty}(\mu)$, then we have extended $\{T(t)\}$ to a Dunford-Schwartz semigroup and $\hat{T}(t) f(x)$ is in the equivalence class of $T(t) f$ for all $t>0$ and $f \in L_{\infty}(\mu)$. One can see that given $f \in L_{\infty}(\mu)$, there exists a $\mu$-null set $E(f)$, independent of $\alpha>0$, outside of which $\int_{0}^{\alpha} \hat{T}(t) f(x) d t$ exists and is finite. If $f \in L_{1}(\mu) \cap L_{\infty}(\mu)$ then $\hat{T}(t) f(x)$ and $T(t) f(x)$ are equivalent scalar representations of $T(t) f$. Finally, if $f=g+h$, where $g \in L_{1}(\mu)$ and $h \in L_{\infty}(\mu)$, we define

$$
T(t) f(x)=\hat{T}(t) g(x)+\hat{T}(t) h(x)
$$

We note that this definition of $T(t) f(x)$ is independent, modulo a $\rho$-null set, of the particular $g$ and $h$ chosen for the representation of $f$.

2. Theorem. Let $(X, \mu)$ be a complete, $\sigma$-finite measure space and let $\left\{T_{k}(t)\right.$ : $t>0\}, k=1,2, \ldots, n$, be strongly continuous $L_{1}(\mu)$ contraction semigroups such that, for all $t \geqslant 0$ and $f \in L_{1}(\mu) \cap L_{\infty}(\mu),\left\|T_{k}(t) f\right\|_{\infty}<\|f\|_{\infty}$. If $f \in L_{p}(\mu)$, $1<p<\infty$, then $A\left(T_{n}, \alpha_{n}\right) \cdots A\left(T_{1}, \alpha_{1}\right) f(x) \rightarrow f(x)$ both in norm and pointwise as $\alpha_{1} \searrow 0, \ldots, \alpha_{n} \searrow 0$ independently.

Proof. By Lemma 1 each $\left\{T_{k}(t)\right\}$ may be regarded as a Dunford-Schwartz semigroup. As pointed out in [2], $\left\{T_{k}(t)\right\}$ is a strongly continuous semigroup of $L_{p}(\mu)$ contractions for $1<p<\infty$. If $f \in L_{p}(\mu)$ and if $f_{k}^{*}(x)$ denotes $\sup _{\alpha>0}\left|(1 / \alpha) \int_{0}^{\alpha} T_{k}(t) f(x) d t\right|$, then $\left\|f_{k}^{*}\right\|_{p} \leqslant(p /(p-1))\|f\|_{p}$ by Theorem VIII.7.7 in [2]. Since $L_{1}(\mu) \cap L_{p}(\mu)$ is dense in $L_{p}(\mu)$ and $\lim _{\alpha_{k} \backslash 0} A\left(T_{k}, \alpha_{k}\right) f(x)=f(x)$ $\mu$-a.e. for all $f \in L_{1}(\mu) \cap L_{p}(\mu)$ by Ornstein's theorem [8, p. 108], it follows from Banach's convergence principle [2, Theorem IV.11.3] that, for each $k$, $\lim _{\alpha_{k} \backslash 0} A\left(T_{k}, \alpha_{k}\right) f(x)$ exists and is finite $\mu$-a.e. as $\alpha_{k} \searrow 0$ through some countable set, say $Q^{+}$, the set of positive rationals. Since $A\left(T_{k}, \alpha_{k}\right) f(x)$ depends continuously on $\alpha_{k}$ for a.e. $x$, we have $\lim _{\alpha_{k} \backslash 0} A\left(T_{k}, \alpha_{k}\right) f(x)$ exists and is finite $\mu$-a.e. for every $f \in L_{p}(\mu)$. The fact that $\lim _{\alpha_{k} \backslash 0} A\left(T_{k}, \alpha_{k}\right) f(x)=f(x) \mu$-a.e. follows from the strong continuity of $\{T(t)\}$ at $t=0$. 
Now consider the convergence of $A\left(T_{n}, \alpha_{n}\right) \cdots A\left(T_{1}, \alpha_{1}\right) f(x)$. Since $\left|A\left(T_{n}, \alpha_{n}\right) \cdots A\left(T_{1}, \alpha_{1}\right) f(x)-f(x)\right|$

$$
\begin{aligned}
& <\sum_{k=1}^{n-1}\left|A\left(T_{n}, \alpha_{n}\right) \cdots A\left(T_{k+1}, \alpha_{k+1}\right)\left[A\left(T_{k}, \alpha_{k}\right) f(x)-f(x)\right]\right| \\
& \quad+\left|A\left(T_{n}, \alpha_{n}\right) f(x)-f(x)\right|,
\end{aligned}
$$

our result is established if we can show

$$
A\left(T_{n}, \alpha_{n}\right) \cdots A\left(T_{k+1}, \alpha_{k+1}\right)\left[A\left(T_{k}, \alpha_{k}\right) f(x)-f(x)\right] \rightarrow 0 \quad \mu \text {-a.e. }
$$

for $k=1,2, \ldots, n-1$. If $\left\{P_{1}(t)\right\}, \ldots,\left\{P_{k}(t)\right\}$ are strongly continuous semigroups of positive Dunford-Schwartz operators which dominate, respectively, $\left\{T_{1}(t)\right\}, \ldots,\left\{T_{n}(t)\right\}$, then

$$
\begin{aligned}
&\left|A\left(T_{n}, \alpha_{n}\right) \cdots A\left(T_{k+1}, \alpha_{k+1}\right)\left[A\left(T_{k}, \alpha_{k}\right) f(x)-f(x)\right]\right| \\
& \leqslant A\left(P_{n}, \alpha_{n}\right) \cdots A\left(P_{k+1}, \alpha_{k+1}\right)\left[\left|A\left(T_{k}, \alpha_{k}\right) f(x)-f(x)\right|\right] .
\end{aligned}
$$

We have

$$
\begin{aligned}
\| A\left(P_{n}, \alpha_{n}\right) \cdots & A\left(P_{k+1}, \alpha_{k+1}\right)\left[A\left(T_{k}, \alpha_{k}\right) f(x)-f(x)\right] \|_{p} \\
& \leqslant((p-1) / p)^{n-k}\left\|A\left(T_{k}, \alpha_{k}\right) f(x)-f(x)\right\|_{p}, \quad k=1,2, \ldots, n-1 .
\end{aligned}
$$

Consequently, given $\varepsilon>0$,

$$
\begin{aligned}
& \mu\left\{\limsup _{\alpha_{1} \succ 0, \ldots, \alpha_{n} \succ 0}\right.\left.\left|A\left(T_{n}, \alpha_{n}\right) \cdots A\left(T_{k+1}, \alpha_{k+1}\right)\left[A\left(T_{k}, \alpha_{k}\right) f(x)-f(x)\right]\right|>\varepsilon\right\} \\
&<\left(\frac{1}{\varepsilon}\right)^{p}\left[\left(\frac{p}{p-1}\right)^{p}\right]^{n-k} \cdot\left\|A\left(T_{k}, \alpha_{k}\right) f(\cdot)-f\right\|_{p}^{p} \text { for any } \alpha_{k}>0 .
\end{aligned}
$$

Since $\left\|A\left(T_{k}, \alpha_{k}\right) f-f\right\|_{p}^{p} \rightarrow 0$ as $\alpha_{k} \searrow 0$ by dominated convergence, we have

$$
A\left(T_{n}, \alpha_{n}\right) \cdots A\left(T_{k+1}, \alpha_{k+1}\right)\left[A\left(T_{k}, \alpha_{k}\right) f(x)-f(x)\right] \rightarrow 0
$$

$\mu$-a.e. as $\alpha_{1} \searrow 0, \ldots, \alpha_{n} \searrow 0$ independently. The norm convergence of $A\left(T_{n}, \alpha_{n}\right) \cdots A\left(T_{1}, \alpha_{1}\right) f$ follows from dominated convergence.

3. THEOREM. Let $\left\{T_{k}(t)\right\}, k=1,2, \ldots, n$, be strongly continuous $L_{1}(\mu)$ contraction semigroups such that $\left\|T_{k}(t) f\right\|_{\infty} \leqslant\|f\|_{\infty}, f \in L_{1}(\mu) \cap L_{\infty}(\mu)$. Then

$$
\lim _{\alpha_{1} \succ 0, \ldots, \alpha_{n} \succ 0} A\left(T_{n}, \alpha_{n}\right) \cdots A\left(T_{1}, \alpha_{1}\right) f(x)=f(x) \quad \mu \text {-a.e. }
$$

for $f \in R_{n-1}$.

Proof. For $f \in R_{n-1}$, set

$$
\begin{aligned}
\omega(f)= & \lim _{\alpha_{1} \succ 0, \ldots, \alpha_{n} \succ 0} A\left(T_{n}, \alpha_{n}\right) \cdots A\left(T_{1}, \alpha_{1}\right) f(x) \\
& -\liminf _{\alpha_{1} \succ 0, \ldots, \alpha_{n} \succ 0} A\left(T_{n}, \alpha_{n}\right) \cdots A\left(T_{1}, \alpha_{1}\right) f(x) .
\end{aligned}
$$

Choose a sequence $f_{k}(k=1,2, \ldots)$ of functions in $L_{p}(\mu), 1<p<\infty$, with $f_{k}(x) \rightarrow f(x) \mu$-a.e. and $\left|f-f_{k}\right| \leqslant|f|$ for all $k$. Then

$$
\omega(f)<\omega\left(f-f_{k}\right)+\omega\left(f_{k}\right)<\omega\left(f-f_{k}\right)
$$


by the preceding theorem. So $\omega(f)<\omega\left(f-f_{k}\right)<2\left(f-f_{k}\right)^{*}$, where

$$
\left(f-f_{k}\right)^{*}(x)=\sup _{\alpha_{1}>0, \ldots, \alpha_{n}>0}\left|A\left(T_{n}, \alpha_{n}\right) \cdots A\left(T_{1}, \alpha_{1}\right)\left(f-f_{k}\right)(x)\right| .
$$

Since the semigroups $\left\{T_{1}(t)\right\}, \ldots,\left\{T_{n}(t)\right\}$ are dominated by positive DunfordSchwartz semigroups, it follows from Fava's dominated estimate [3] that

$$
\begin{aligned}
\mu\{\omega(f)>8 t\} & \leqslant \mu\left\{\left(f-f_{k}\right)^{*}>4 t\right\} \\
& \leqslant C_{n-1} \int_{\left|f-f_{k}\right|>t}\left|\frac{f-f_{k}}{t}\right|\left(\log \left|\frac{f-f_{k}}{t}\right|\right)^{n-1} d \mu \quad \text { for any } t>0 .
\end{aligned}
$$

The integral approaches zero as $k \rightarrow \infty$ by dominated convergence. Thus $\mu\{\omega(f)>$ $8 t\}=0$ for all $t>0$, and so $\lim _{\alpha_{1}>0, \ldots, \alpha_{n}>0} A\left(T_{n}, \alpha_{n}\right) A\left(T_{n-1}, \alpha_{n-1}\right) \cdots$ $A\left(T_{1}, \alpha_{1}\right) f(x)$ exists and is finite $\mu$-a.e. Setting

$$
\tilde{f}(x)=\lim _{\alpha_{1}>0, \ldots, \alpha_{n}>0} A\left(T_{n}, \alpha_{n}\right) \cdots A\left(T_{1}, \alpha_{1}\right) f(x),
$$

we have

$$
\begin{aligned}
\mu\left\{\left(\tilde{f}-f_{k}\right)>4 t\right\} & =\mu\left\{\lim _{\alpha_{1}>0, \ldots, \alpha_{n}>0} A\left(T_{n}, \alpha_{n}\right) \cdots A\left(T_{1}, \alpha_{1}\right)\left(f-f_{k}\right)>4 t\right\} \\
& <\mu\left\{\left(f-f_{k}\right)^{*}>4 t\right\} \\
& \leqslant C_{n-1} \int_{\left|f-f_{k}\right|>t}\left|\frac{f-f_{k}}{t}\right|\left(\log \left|\frac{f-f_{k}}{t}\right|\right)^{n-1} d \mu .
\end{aligned}
$$

Likewise

$$
\mu\left\{\left(f_{k}-\tilde{f}\right)>4 t\right\}<C_{n-1} \int_{\left|f-f_{k}\right|>t}\left|\frac{f-f_{k}}{t}\right|\left(\log \left|\frac{f-f_{k}}{t}\right|\right)^{n-1} d \mu .
$$

These two inequalities imply $f_{k} \rightarrow \tilde{f}$ in $\mu$-measure. By Corollary III.6.13 in [2], there exists a subsequence $\left\{f_{n_{k}}\right\}$ which converges to $\tilde{f} \mu$-a.e. Since $f_{k} \rightarrow f$ pointwise, we must have $\tilde{f}(x)=f(x) \mu$-a.e.

Since $L_{1}(\mu)$ is strictly contained in $R_{0}$, the preceding theorem for the case $n=1$ yields a slight generalization of Ornstein's local ergodic theorem [8].

\section{REFERENCES}

1. M. Akcoglu and R. Chacon, A local ratio theorem, Canad. J. Math. 22 (1970), 545-552.

2. N. Dunford and J. T. Schwartz, Linear operators. I, Interscience, New York, 1958.

3. N. Fava, Weak type inequalities for product operators, Studia Math. 42 (1972), 271-288.

4. C. Kipnis, Majoration des semi-groupes de contractions de $L_{1}$ et applications, Ann. Inst. H. Poincaré Sect. B 10 (1974), 369-384.

5. U. Krengel, A local ergodic theorem, Invent. Math. 6 (1969), 329-333.

6. Y. Kubokawa, Ergodic theorems for contraction semigroups, J. Math. Soc. Japan 27 (1975), 184-193.

7. J. Neveu, Mathematical foundations of the calculus of probability, Holden-Day, San Francisco, Calif., 1965.

8. D. Ornstein, The sums of iterates of a positive operator, Advances in Probability and Related Topics (P. Ney, ed.), vol. 2, Dekker, New York, 1970, pp. 87-115.

9. R. Sato, On a local ergodic theorem, Studia Math. 58 (1976), 1-5.

10. T. Terrell, Local ergodic theorems for n-parameter semigroups of operators, Lecture Notes in Math., vol. 160, Springer-Verlag, Berlin and New York, 1970, pp. 262-278.

Sperry Systems Management, Great Neck, New York 11020 